hep-th/0606178

DAMTP-2006-51

\title{
Two universal results for Wilson loops at strong coupling
}

\author{
Sean A. Hartnoll \\ DAMTP, Centre for Mathematical Sciences, Cambridge University \\ Wilberforce Road, Cambridge CB3 OWA, UK \\ s.a.hartnoll@damtp.cam.ac.uk
}

\begin{abstract}
We present results for Wilson loops in strongly coupled gauge theories. The loops may be taken around an arbitrarily shaped contour and in any field theory with a dual IIB geometry of the form $M \times S^{5}$. No assumptions about supersymmetry are made. The first result uses D5 branes to show how the loop in any antisymmetric representation is computed in terms of the loop in the fundamental representation. The second result uses D3 branes to observe that each loop defines a rich sequence of operators associated with minimal surfaces in $S^{5}$. The action of these configurations are all computable. Both results have features suggesting a connection with integrability.
\end{abstract}




\section{Introduction}

Wilson loop operators are basic nonlocal gauge invariant operators in any Yang-Mills theory. In the AdS/CFT correspondence [1, Wilson loops are described by similarly basic objects in string theory. The original strong coupling dual description of such operators was in terms of noncompact worldsheet instantons in the bulk geometry ending on the loop at conformal infinity [2, 3]. There has been a recent revival of interest in Wilson loops in the AdS/CFT correspondence. Of particular relevance to the present work is the appreciation that probe D3 and D5 branes are also important for computing certain Wilson loop observables [4, 5, 6, 7, 8, 9, 10, 11].

Much of the tangible progress to date in studying Wilson loop operators in the AdS/CFT correspondence has stemmed from the fact that certain loops are BPS operators of the $\mathcal{N}=4$ super Yang-Mills (SYM) theory. The works of [12, 13] have argued that half BPS circular Wilson loops are computed exactly, up to instanton corrections [14, to all orders in $1 / N$ and $\lambda$ by a quadratic Hermitian matrix model. This observation has allowed some of the most precise tests yet of the AdS/CFT duality [4, 17, 10, 11].

The results presented in this letter are universal in the sense that they apply to a very wide range of Wilson loops at strong coupling and do not depend on supersymmetry. Firstly, they are applicable to Wilson loops in any gauge theory with a dual IIB geometry of the form $M \times S^{5}$. The manifold $M$ can have any asymptotically (locally) AdS five dimensional metric with constant negative Ricci tensor. It could be $A d S_{5}$, but it could also be, for instance, the five dimensional AdS-Schwarzschild black hole. Therefore all our results apply to Wilson loops at finite temperature. Secondly, the results are applicable to Wilson loops where the $S U(N)$ holonomy is taken around an arbitrary curve in the boundary field theory. Such a loop will generically not preserve any supercharges.

Our first result, in section 2, shows that the expectation value of a Wilson loop in the rank $k$ antisymmetric representation, computed by a D 5 brane with $k$ units of worldvolume flux [7, 8, 11, is given by a universal expression in terms of the loop in the fundamental representation, computed by a fundamental string. The relation is naturally expressed in terms of a Hermitian matrix model. This is surprising given that we are a long way away from BPS loops.

Section 3 is a warm up for our second result, in section 4 . We observe that for every Wilson loop with a dual string $\Sigma \hookrightarrow M$, there is an infinite sequence of D3 brane immersions $\Sigma \times \Upsilon \hookrightarrow M \times S^{5}$, where $\Upsilon$ is a minimal surface in $S^{5}$. These configurations have a rich purely algebraic description as holomorphic curves in $\mathbb{C P}^{4}$, and their actions are all computable. 


\section{A universal result for antisymmetric loops}

Consider a general IIB background of the form $M \times S^{5}$. We can write the metric as

$$
d s^{2}=R^{2}\left[d s_{M}^{2}+d \theta^{2}+\sin ^{2} \theta d \Omega_{S^{4}}^{2}\right],
$$

where the polar angle $\theta \in[0, \pi]$. Suppose we have an arbitrary Wilson loop in the boundary theory that preserves $S O(5) \subset S O(6)_{R}$, that is to say, a loop in $\partial M$ times a point in $S^{5}$. It is well known that if the loop is in the fundamental representation, then the state dual to this operator is a fundamental string embedded on a minimal surface $\Sigma \subset M$ that ends on the loop in $\partial M$. We now derive a universal expression for a Wilson loop on the same curve on the boundary but in the rank $k$ antisymmetric representation.

Wilson loops in the $k$-th antisymmetric representation are dual to a D5 brane embedded in the dual geometry carrying $k$ units of worldvolume flux [7, 8, 11]. Recall that the Euclidean D5 brane action is

$$
S_{D 5}=T_{D 5} \int d \tau d^{5} \sigma \sqrt{\operatorname{det}\left({ }^{\star} g+2 \pi \alpha^{\prime} F\right)}-i g_{s} T_{D 5} \int 2 \pi \alpha^{\prime} F \wedge{ }^{\star} C_{4},
$$

where $T_{D 5}=N \sqrt{\lambda} / 8 \pi^{4} R^{6}$. Here and elsewhere we freely use the relation $R^{4}=\lambda \alpha^{\prime 2}$. The relevant part of the background four form potential is

$$
C_{4}=\frac{R^{4}}{g_{s}}\left[\frac{3(\theta-\pi)}{2}-\sin ^{3} \theta \cos \theta-\frac{3}{2} \cos \theta \sin \theta\right] \operatorname{vol} S^{4},
$$

where $\theta$ is a polar angle in the $S^{5}$, as in (1).

The D5 brane embedding we are looking for will be $\Sigma \times S^{4} \hookrightarrow M \times S^{5}$. The $S^{4}$ is set to be at an angle $\theta$ in the $S^{5}$. This embedding preserves an $S O(5)$ subgroup of the R symmetry, as did the original worldsheet $\Sigma$. Furthermore, in order to induce a string charge, we need to have an electric worldvolume gauge field. This field will be imaginary because we need to set $\delta S / \delta F_{\tau \sigma}=i k$ to carry the charge of $k \in \mathbb{Z}$ fundamental strings. Thus we set

$$
F_{\tau \sigma} \equiv i F \frac{\sqrt{\lambda}}{2 \pi} .
$$

In these expressions we are using $\tau, \sigma$ as coordinates on the surface $\Sigma$. The action for the D5 brane on this ansatz becomes

$$
S_{D 5}=\frac{N \sqrt{\lambda}}{3 \pi^{2}} \int d \tau d \sigma\left[\sin ^{4} \theta \sqrt{\operatorname{det}^{*} g_{\Sigma}-F^{2}}-D(\theta) F\right],
$$


where we have introduced

$$
D(\theta)=\sin ^{3} \theta \cos \theta+\frac{3}{2} \cos \theta \sin \theta-\frac{3(\theta-\pi)}{2} .
$$

In the action (5),${ }^{*} g_{\Sigma}$ denotes the metric pulled back from $M \times S^{5}$, with all factors of $R$ stripped off, onto the worldsheet $\Sigma$.

The equations of motion for $\theta$ and $F$ allow for constant solutions $\theta=\theta_{0}$, provided that $\theta_{0}$ satisfies

$$
\pi\left(\frac{k}{N}-1\right)=\sin \theta_{0} \cos \theta_{0}-\theta_{0}
$$

For these solutions,

$$
F=-\cos \theta_{0} \sqrt{\operatorname{det}^{*} g_{\Sigma}} .
$$

Thus as a two form, $F$ is proportional to the volume form on $\Sigma$. Using the constancy of $\theta$ and the above expression for $F$, it is straightforward to show that the equations of motion for the embedding $X^{\mu}\left(\sigma_{a} \equiv\{\tau, \sigma\}\right)$ into $M$ become

$$
\frac{\partial \sqrt{\operatorname{det}^{*} g_{\Sigma}}}{\partial X^{\mu}}=\partial_{a} \frac{\partial \sqrt{\operatorname{det}^{*} g_{\Sigma}}}{\partial \partial_{a} X^{\mu}}
$$

Note that $\theta$ being constant implies that ${ }^{*} g_{\Sigma}$ only depends on the embedding into $M$ and not on $S^{5}$. The equations (9) are of course precisely the equations of motion following from the Nambu-Goto string action

$$
S_{F 1}=\frac{1}{2 \pi \alpha^{\prime}} \int d \tau d \sigma \sqrt{\operatorname{det}^{\star} g_{\Sigma}}
$$

Therefore, we have found that any embedding $\Sigma$ into $M$ of a fundamental string defines an embedding $\Sigma \times S^{4}$ of a D5 brane into $M \times S^{5}$ for every $k \in \mathbb{Z}$. Now we need to compute their actions.

In evaluating the action of the D5 brane on the solution, we need to add the following boundary terms. These may be thought of as implementing the correct Neumann and Dirichlet boundary conditions and infinity and simultaneously renormalising the action [15, 4, 6]. We have

$$
\left.S_{D 5}\right|_{\mathrm{bdy} .}=-\int_{\partial \Sigma} d \tau X^{\mu} \frac{\delta S_{D 5}}{\delta \partial_{\sigma} X^{\mu}}-\int_{\partial \Sigma} d \tau \theta \frac{\delta S_{D 5}}{\delta \partial_{\sigma} \theta}+\frac{\sqrt{\lambda}}{2 \pi} \int_{\Sigma} d \tau d \sigma k F
$$

In this expression we have taken $\tau$ to be the worldvolume coordinate tangent to the boundary and $\sigma$ the worldvolume coordinate normal to the boundary. The first of these terms is the same as that required to renormalise the fundamental string action. The 
second in fact vanishes on the solution. Using (8) and constancy of $\theta$ we can evaluate the total action to obtain

$$
\left.S_{D 5}\right|_{\text {renor. }}=\left.\frac{2 N}{3 \pi} \sin ^{3} \theta_{0} S_{F 1}\right|_{\text {renor. }} \cdot
$$

Therefore we find that for a very wide class of Wilson loops, independently of the five dimensional background and of the shape of the loop, the loop in the $k$-th antisymmetric representation is given in terms of the loop in the fundamental representation through the formula (12), with $\theta_{0}$ given by (7). This implies, for example, that antisymmetric loops have exactly the same dependence on $k$ at zero and finite temperature.

The existence of probe D5 brane embeddings at the angles (17) determined by worldvolume flux has been observed in various different contexts, see for instance [16, 17, 18, 6, 7]. The expression (12) is a substantial generalisation of the known results, albeit technically straightforward. An especially striking aspect of (12) is that it is the result one obtains for the antisymmetric loop in a quadratic Hermitian matrix model [1]. That is to say, (12) can be rewritten as the $N \rightarrow \infty, S_{F 1} \gg 1$ limit of

$$
e^{-S_{D 5, k}}=\int[d M] \operatorname{Tr}_{A_{k}}\left[e^{M}\right] e^{-2 N / S_{F 1}^{2} \operatorname{Tr}\left[M^{2}\right]} .
$$

In this last expression both the D5 and F1 actions are understood to be renormalised. The $A_{k}$ index indicates that the trace is taken in the rank $k$ totally antisymmetric representation of $S U(N)$. The matrix $M$ is an $N \times N$ Hermitian matrix.

For the circular BPS Wilson loop, $S_{F 1}=-\sqrt{\lambda}$ and one recovers the expected matrix model of [12, 13]. The appearance of a matrix model in a much more general setting clearly raises many interesting questions. We should emphasise, however, that we have only made the connection in the large $N$, large $\lambda$ limit. There may be many matrix model and non matrix model computations other than (13) that lead to the same answer (12) in that limit.

\section{Some D3 brane embeddings}

One might search for a similar universal result for Wilson loops in the rank $k$ symmetric representation. These are probably dual to a D3 brane in the bulk geometry [8, 11]. This appears to be a harder question than the antisymmetric case and we will not address it here. Instead, we describe some intriguing D3 brane solutions that are related to Wilson loops.

The reason that the symmetric representations are difficult is the following. We expect the worldvolume flux and background Ramond-Ramond potential to blow up the 
worldsheet from $\Sigma$ to $\Sigma \times S^{2}$. However, in order to preserve the $S O(5)$ R-symmetry of the Wilson loop, the whole $\Sigma \times S^{2}$ worldvolume should be embedded into $M$ rather than $M \times S^{5}$. The required D3 brane solution has been found for the case of a circular loop when $M=A d S_{5}$ 4. However, for more general loops, or more general backgrounds with less or no symmetry, finding the embedding appears to be a daunting task that needs to be approached on a case by case basis.

In this section and the next we will look for D3 brane solutions which are similar to the D5 brane solutions of the previous section. They will be the product of a string embedding $\Sigma$ into $M$ and a map from $S^{2}$ into $S^{5}$. To begin with we require $S^{2}$ to be embedded into $S^{5}$. We then relax this restriction to allow self intersections, revealing a rich mathematical structure underpinning the solutions.

Let us write the background metric as follows

$$
d s^{2}=R^{2}\left[d s_{M}^{2}+d \alpha^{2}+\sin ^{2} \alpha d \Omega_{S^{2}}^{2}+\cos ^{2} \alpha d \widetilde{\Omega}_{S^{2}}^{2}\right],
$$

where $\alpha \in[0, \pi / 2]$. Given an arbitrary fundamental string embedding of $\Sigma$ into $M$, we will show in this section that there is a D3 embedding of $\Sigma \times S^{2} \hookrightarrow M \times S^{5}$ carrying $k$ units of worldvolume flux. This embedding will break the $S O(5)$ symmetry of $\Sigma$ down to $S O(3) \times S O(2)$.

The relevant D3 brane action is

$$
S_{D 3}=T_{D 3} \int d \tau d^{3} \sigma \sqrt{\operatorname{det}\left({ }^{\star} g+2 \pi \alpha^{\prime} F\right)},
$$

where the tension $T_{D 3}=N / 2 \pi^{2} R^{4}$. We have not written down the Wess-Zumino term as this will not contribute to the configurations under consideration, which have two directions in $M$ and two directions in $S^{5}$. More precisely, the embedding we look for will wrap the first $S^{2}$ in (14) at some value of $\alpha$. As before, we set

$$
F_{\tau \sigma} \equiv i F \frac{\sqrt{\lambda}}{2 \pi} .
$$

The action for the D3 brane becomes

$$
S_{D 3}=\frac{2 N}{\pi} \int d \tau d \sigma \sin ^{2} \alpha \sqrt{\operatorname{det}^{*} g_{\Sigma}-F^{2}} .
$$

Here again, ${ }^{*} g_{\Sigma}$ denotes the metric pulled back from the embedding onto $\Sigma$.

The equation of motion for $\alpha$ allows for two constant solutions, $\alpha=\alpha_{0}=0$ and $\alpha=\alpha_{0}=\pi / 2$. The first of these corresponds to the $S^{2}$ collapsing to a point and takes us outside the regime of validity of the Dirac-Born-Infeld action (15). The second is 
fully reliable and non collapsed. On these solutions, the equation of motion for $F$ may be solved to give

$$
F=\frac{\kappa}{\sqrt{\sin ^{4} \alpha_{0}+\kappa^{2}}} \sqrt{\operatorname{det}^{*} g_{\Sigma}} .
$$

Once again, the worldvolume gauge field is proportional to the volume form on the worldsheet. As is usual in D3 brane computations [4, 6], we have introduced

$$
\kappa=\frac{\sqrt{\lambda} k}{4 N}
$$

It is then immediate to check that the embedding of $\Sigma$ into $M$ must satisfy the equations following from the Nambu-Goto action (10). Therefore, for any embedding of a fundamental string into $M$ we have found an embedding of a probe D3 brane into $M \times S^{5}$ for all $k \in \mathbb{Z}$.

To renormalise the action and impose the correct boundary conditions at infinity, we need to add the boundary term

$$
\left.S_{D 3}\right|_{\mathrm{bdy} .}=-\int_{\partial \Sigma} d \tau X^{\mu} \frac{\delta S_{D 3}}{\delta \partial_{\sigma} X^{\mu}}-\int_{\partial \Sigma} d \tau \alpha \frac{\delta S_{D 3}}{\delta \partial_{\sigma} \alpha}+\frac{2 N}{\pi} \int_{\Sigma} d \tau d \sigma \kappa F
$$

The effects of these terms are similar to what occurred in the D5 brane case. The renormalised action becomes

$$
\left.S_{D 3}\right|_{\text {renor. }}=\left.\frac{4 N}{\sqrt{\lambda}} \sqrt{\sin ^{4} \alpha_{0}+\kappa^{2}} S_{F 1}\right|_{\text {renor. }} \cdot
$$

If the renormalised F1 action is negative, as it is for the cases that have been computed, then the blown up solution $\alpha_{0}=\pi / 2$ is favoured over the collapsed solution $\alpha_{0}=0$. Assuming negativity of the F1 action, (21) may be suggestively rewritten as

$$
S_{D 3, k}=-\sqrt{\left(k S_{F 1}\right)^{2}+\left(S_{D 3,0}\right)^{2}}
$$

with all actions in this expression understood to be renormalised.

We can see from (21) that as $k / N \rightarrow 0$ the action does not tend to that of $k$ fundamental strings, suggesting that these solutions should not be understood as blown up fundamental strings. Indeed, the 'blown up' solution remains favoured over the collapsed solution even when $k=0$. Independently of $k$, and because $F$ is proportional to the volume form on $\Sigma$, the D3 branes are simply minimal surfaces in $M \times S^{5}$. Such brane configurations usually have negative modes due to the fact that the $S^{2}$ is contractible within $S^{5}$. However, the renormalised area of $\Sigma$ in $M$ is negative for all known solutions when $M$ is $A d S_{5}$ or Schwarzschild- $A d S_{5}$. Therefore minimising the action (21) favours larger areas of $S^{2} \subset S^{5}$ and the collapsing modes increase rather than decrease the action. This counterintuitive behaviour seems to be related to the imposition of Neumann rather than Dirichlet boundary conditions at infinity for certain of the embedding fields. 


\section{$4 \quad$ D3 brane immersions and minimal surfaces in $S^{5}$}

It becomes clear at this point that we need not have restricted our D3 brane configurations to such a symmetric ansatz. Consider any F1 embedding $\Sigma \hookrightarrow M$, dual to a fundamental representation Wilson loop in the gauge theory. Given any minimal two dimensional surface $\Upsilon$ in $S^{5}$ then $\Sigma \times \Upsilon \hookrightarrow M \times S^{5}$ gives a solution to the D3 brane equations of motion, again for any $k \in \mathbb{Z}$. A straightforward adaptation of the argument in the previous section shows that the action of this configuration is

$$
\left.S_{D 3}\right|_{\text {renor. }}=\left.\frac{4 N}{\sqrt{\lambda}} \sqrt{(\operatorname{Vol} \Upsilon / 4 \pi)^{2}+\kappa^{2}} S_{F 1}\right|_{\text {renor. }} \cdot
$$

As well as the high symmetry, a special feature of the solution of the previous section is that it was an embedding of $S^{2}$ into $S^{5}$. Generic minimal surfaces are not embeddings, but rather they are immersions. That is to say, they self intersect. If we allow the D3 brane to self intersect, then the solution we described in the previous section is just the tip of an iceberg. There does not appear to be any reason to discard self intersecting immersions. It is likely that $\alpha^{\prime}$ corrections will disconnect the brane at the intersection by condensing a tachyonic mode, but at a classical level the self intersecting solution provides an adequate description.

Let us restrict ourselves to the case that $\Upsilon$ is a minimal immersion of $S^{2}$ into $S^{5}$. Higher genus immersions are certainly interesting, but we wish to make use here of the highly developed mathematical theory of immersions of spheres into spheres. The following theorems are applicable to such immersions. A review of this material and some additional theorems can be found, for instance, in [19].

Theorem 1 (Calabi [20], Barbosa [22]) The minimal immersion lies in an extremal $S^{4} \subset S^{5}$. Either it is a totally geodesic $S^{2}$ with area $4 \pi$ or it has area $4 \pi m$, with $m \in \mathbb{Z}$ and $m \geq 3$.

The totally geodesic $S^{2}$ s are the cases that we considered in the previous section. This theorem implies that we can set $\operatorname{Vol} \Upsilon / 4 \pi=m$ in (23). Thus the actions of these configurations are all given in terms of the fundamental string action and an integer, without needing to know the precise form of $\Upsilon$. Before moving on to a general description, note that the cases in which the induced metric on $\Upsilon$ has constant curvature can be characterised very explicitly:

Theorem 2 (Calabi [20]) If the induced metric on $\Upsilon$ has constant curvature, then $\Upsilon$ is either a totally geodesic $S^{2}$ or it is the Veronese surface. 
The Veronese surface in this context is the immersion of $\mathbb{R}^{3}$ into $\mathbb{R}^{6}$

$$
\left(x_{1}, x_{2}, x_{3}\right) \rightarrow\left(\sqrt{3} x_{1} x_{2}, \sqrt{3} x_{1} x_{3}, \sqrt{3} x_{2} x_{3}, \frac{\sqrt{3}}{2}\left(x_{1}^{2}-x_{2}^{2}\right), \frac{1}{2}\left(x_{1}^{2}+x_{2}^{2}-2 x_{3}^{2}\right), 0\right) .
$$

When restricted to $x_{1}^{2}+x_{2}^{2}+x_{3}^{2}=1$, this gives a minimally immersed $S^{2}$ in $S^{4} \subset S^{5}$ with constant curvature and area $12 \pi$, corresponding to $m=3$ in Theorem 1 . Of course, this surface and all immersions are defined up to rigid $S O(6)$ rotations on $S^{5}$.

We now describe general minimal immersions. From Theorem 1 it is sufficient to consider maps $x: S^{2} \rightarrow S^{4}$. We will describe $x$ as a unit vector in $\mathbb{R}^{5}$. We can always choose isothermal coordinates $\{z, \bar{z}\}$ on $S^{2}$ so that the induced metric is

$$
d s_{\Upsilon}^{2}=2 \partial x \cdot \bar{\partial} x d z d \bar{z}
$$

Here and below $a \cdot b$ denotes the symmetric dot product on $\mathbb{C}^{5}$, inherited from $\mathbb{R}^{5}$. With these coordinates, minimal immersions are those that satisfy the nonlinear equation

$$
\partial \bar{\partial} x=-\partial x \cdot \bar{\partial} x x
$$

Remarkably, this equation can be solved in full generality for immersions of $S^{2}$ into $S^{4}$.

Theorem 3 (Chern [21], Barbosa [22]) There is a canonical one to one correspondence between minimal immersions $x: S^{2} \rightarrow S^{4}$ and the set of totally isotropic holomorphic curves $\xi: S^{2} \rightarrow \mathbb{C P}^{4}$.

The general minimal immersion is constructed as follows. Let $\xi$ be homogeneous coordinates on $\mathbb{C P}^{4}$. The holomorphic curves are necessarily polynomials, so that

$$
\xi(z)=\sum_{i=0}^{n} A_{i} z^{i},
$$

with $A_{i} \in \mathbb{C}^{5}$. Total isotropy is the statement that the polynomial must satisfy

$$
\xi \cdot \xi=\partial \xi \cdot \partial \xi=0
$$

Given such a polynomial we define, using roman indices running from 1 to 5 ,

$$
\psi_{a}=\epsilon_{a b c d e} \xi_{b} \partial \xi_{c} \bar{\xi}_{d} \bar{\partial} \bar{\xi}_{e}
$$

It is clear that $\psi \in \mathbb{R}^{5}$. The minimal immersion is then given by

$$
x=\frac{\psi}{|\psi|} .
$$


For example, the following curve in $\mathbb{C P}^{4}$ describes the Veronese immersion (24)

$$
\begin{gathered}
\xi_{1}=2 \sqrt{3}\left(1+z^{4}\right), \quad \xi_{2}=2 \sqrt{3} i\left(1-z^{4}\right), \quad \xi_{3}=-12 z^{2}, \\
\xi_{4}=-4 \sqrt{3} i\left(z+z^{3}\right), \quad \xi_{5}=-4 \sqrt{3}\left(z-z^{3}\right) .
\end{gathered}
$$

Substituting this expression into (29) and (301) and changing the coordinates on $S^{2}$ to $z=\left(x_{1}+i x_{2}\right) /\left(1+x_{3}\right)$ with $x_{1}^{2}+x_{2}^{2}+x_{3}^{2}=1$, one recovers the immersion (24). Using this formalism, Barbosa has constructed examples of regular immersions with all values of the area $4 \pi m$ allowed by Theorem 1 [22]. That paper also contains other interesting results on the moduli space of immersions and their regularity. Minimal immersions in $S^{4}$ can also be described using the Penrose twistor transform. In that description they are given by holomorphic curves in $\mathbb{C P}^{3}$ that are horizontal with respect to the twistor fibration over $S^{4}[23$.

The point we wish to emphasise following from the above discussion is this: to every Wilson loop operator of the boundary theory, with dual F1 embedding $\Sigma \hookrightarrow M$, we can associate an infinite sequence of distinct Wilson loop operators, one for each minimal surface $\Upsilon \hookrightarrow S^{5}$. These new operators generically preserve no R symmetry. Nonetheless, the action of the dual D3 solutions is determined entirely in terms of the original F1 action and a single integer $m$. A priori, each operator is associated with a solution to a nonlinear partial differential equation. However, we have seen that in fact they are counted by the purely algebraic problem of finding totally isotropic holomorphic curves on $\mathbb{C P}^{4}$.

What are these additional operators? We will not attempt to answer this question here. Note that Wilson loop operators can be obtained by integrating any adjoint valued operator around a closed contour $C$ in spacetime. Using only the six adjoint scalars $\left\{\Phi_{I}\right\}$ of the $\mathcal{N}=4 \mathrm{SYM}$ theory, a natural set of operators to consider are

$$
W[C, \Upsilon]=\operatorname{Tr} \mathcal{P} \exp \left[i \oint_{C} d s\left(A(s)+i \Theta_{I} \Phi_{I}(s)+\sum_{n \geq 2} f_{I_{1} \cdots I_{n}} \Phi_{I_{1}}(s) \cdots \Phi_{I_{n}}(s)\right)\right],
$$

with coefficients $f_{I_{1} \cdots I_{n}}$ defined in terms of integrals over the immersion $\Upsilon$. In this expression $A$ is the $S U(N)$ gauge field. The first two terms in the exponent are those appearing in the usual Euclidean Maldacena-Wilson loop of the $\mathcal{N}=4$ theory. For the loops we have been considering, with $\Sigma \hookrightarrow M, \Theta^{I}$ is a constant unit vector in $\mathbb{R}^{6}$. A simple possibility for the first coefficient beyond the standard term is

$$
f_{I J}=\int_{\Upsilon} \sqrt{\operatorname{det}^{*} g_{\Upsilon}} g_{\Upsilon}^{i j} \partial_{i} x_{I} \partial_{j} x_{J}=\int_{\Upsilon} d z d \bar{z} \partial x_{I} \bar{\partial} x_{J},
$$

where $x_{I}(z, \bar{z})$ is the immersion of $\Upsilon$ into $S^{5} \subset \mathbb{R}^{6}$ and ${ }^{*} g_{\Upsilon}$ is the induced metric (25). The definition of these operators should not require $\Upsilon$ to be a minimal surface. When 
the surface is minimal, however, we have seen that the operators have a particularly simple dual description. The elegant description of this class of Wilson loop operators at strong coupling clearly warrants a field theory understanding.

\section{Concluding remarks}

We have not yet pursued a field theory understanding of the results in this letter. The possible appearance of a matrix model in the study of antisymmetric representations, and of an integrable nonlinear partial differential equation underpinning the D3 brane configurations, suggests that perhaps similar integrable structures should be present in the field theory duals.

For the case of the D3 brane configurations, it is important to understand whether there are normalisable negative modes about the solutions. The minimal immersions $\Upsilon \hookrightarrow S^{5}$ define the set of possible asymptotic configurations near the boundary. However, if there is a negative mode then the dominant contribution to the expectation value will come from a more complicated configuration than $\Sigma \times \Upsilon$. It would also be interesting to understand the case when $\Upsilon$ is of higher genus.

\section{Acknowledgements}

I would like to thank Gary Gibbons, Nick Dorey and especially Prem Kumar for helpful comments. The author is supported by a research fellowship from Clare college, Cambridge.

\section{References}

[1] J. M. Maldacena, "The large $N$ limit of superconformal field theories and supergravity," Adv. Theor. Math. Phys. 2, 231 (1998) [Int. J. Theor. Phys. 38, 1113 (1999)] arXiv:hep-th/9711200.

[2] J. M. Maldacena, "Wilson loops in large N field theories," Phys. Rev. Lett. 80 (1998) 4859 arXiv:hep-th/9803002.

[3] S. J. Rey and J. T. Yee, "Macroscopic strings as heavy quarks in large N gauge theory and anti-de Sitter supergravity," Eur. Phys. J. C 22 (2001) 379 arXiv:hep-th/9803001. 
[4] N. Drukker and B. Fiol, "All-genus calculation of Wilson loops using D-branes," JHEP 0502 (2005) 010 arXiv:hep-th/0501109.

[5] S. Yamaguchi, "Bubbling geometries for half BPS Wilson lines," arXiv:hep-th/0601089.

[6] S. A. Hartnoll and S. Prem Kumar, "Multiply wound Polyakov loops at strong coupling," arXiv:hep-th/0603190.

[7] S. Yamaguchi, "Wilson loops of anti-symmetric representation and D5-branes," arXiv:hep-th/0603208.

[8] J. Gomis and F. Passerini, "Holographic Wilson Loops," arXiv:hep-th/0604007.

[9] O. Lunin, "On gravitational description of Wilson lines," arXiv:hep-th/0604133.

[10] K. Okuyama and G. W. Semenoff, "Wilson Loops in N=4 SYM and Fermion Droplets," arXiv:hep-th/0604209.

[11] S. A. Hartnoll and S. P. Kumar, "Higher rank Wilson loops from a matrix model," arXiv:hep-th/0605027.

[12] J. K. Erickson, G. W. Semenoff and K. Zarembo, "Wilson loops in N = 4 supersymmetric Yang-Mills theory," Nucl. Phys. B 582 (2000) 155 arXiv:hep-th/0003055.

[13] N. Drukker and D. J. Gross, "An exact prediction of N $=4$ SUSYM theory for string theory," J. Math. Phys. 42 (2001) 2896 arXiv:hep-th/0010274.

[14] M. Bianchi, M. B. Green and S. Kovacs, "Instanton corrections to circular Wilson loops in N = 4 supersymmetric Yang-Mills," JHEP 0204, 040 (2002) arXiv:hep-th/0202003.

[15] N. Drukker, D. J. Gross and H. Ooguri, "Wilson loops and minimal surfaces," Phys. Rev. D 60 (1999) 125006 arXiv:hep-th/9904191.

[16] C. G. . Callan, A. Guijosa and K. G. Savvidy, "Baryons and string creation from the fivebrane worldvolume action," Nucl. Phys. B 547 (1999) 127 arXiv:hep-th/9810092.

[17] J. M. Camino, A. V. Ramallo and J. M. Sanchez de Santos, "Worldvolume dynamics of D-branes in a D-brane background," Nucl. Phys. B 562 (1999) 103 arXiv:hep-th/9905118.

[18] J. Gomis, P. K. Townsend and M. N. R. Wohlfarth, "The 's-rule' exclusion principle and vacuum interpolation in worldvolume dynamics," JHEP 0212 (2002) 027 arXiv:hep-th/0211020.

[19] H.B.Lawson, "Minimal varieties," Proc. Sympos. Pure Math. 27 (1975) 143.

[20] E. Calabi, "Minimal immersions of surfaces in Euclidean spheres," J. Diff. Geom. 1 (1967) 111. 
[21] S-S. Chern, "On minimal spheres in the four-sphere," in Studies and Essays (Presented to Yu-why Chen on his 60th Birthday), pp. 137 - 150, Math. Res. Center, Nat. Taiwan Univ., Taipei (1970).

[22] J.L.M. Barbosa, "On minimal immersions of $S^{2}$ into $S^{2 m}$," Trans. Amer. Math. Soc. 210 (1975) 75.

[23] R.L. Bryant, "Conformal and minimal immersions of compact surfaces into the 4-sphere," J. Diff. Geom. 17 (1982) 455. 